\title{
Nivel de estrés en estudiantes de medicina de la Universidad Santa Clara de Asís, Paraguay
}

\author{
Luz Diana Paredes Rojas ${ }^{1}$, Sirley Patricia Paniagua Rojas ${ }^{1}$
}

\begin{abstract}
Resumen
Introducción: Podemos definir al estrés como un proceso de índole psicosocial en el que no sólo ha de tenerse en cuenta la respuesta fisiológica del organismo, sino que como ya mencionamos, las características del ambiente social y factores personales. Estas variables moduladoras determinan la percepción de estrés y sus efectos sobre la salud y el bienestar e inciden desde la aparición de los factores causales del estrés hasta las consecuencias, por lo que contribuyen al grado de éxito con el que se afronta el estresor y las demandas ambientales. En este sentido, destacamos estudiantes de medicina que también son vulnerables a desarrollar alteraciones de la salud mental por causas como entornos estresantes a los que son sometidos diariamente (situación económica, imposiciones de carga emocional), un ritmo de vida al cual no estaban acostumbrados (largas horas de estudios, falta de descanso adecuado) o condiciones familiares (personas con las que se vive, nivel de educación alcanzado por los padres) que actúan como factores perjudiciales para el completo bienestar. Ante el estrés académico, los estudiantes deben dominar cada vez más las exigencias y retos que les demanda la gran cantidad de recursos físicos y psicológicos para enfrentar esta problemática.
\end{abstract}

Objetivo: Determinar el nivel de estrés académico que presentan los estudiantes de medicina de la "Universidad Santa Clara de Asís" de Caaguazú, año 2019.

Métodos: Estudio observacional, descriptivo y transversal con una muestra no probabilística de estudiantes del 30 al 6o curso del primer semestre del año 2019, tomadas de manera casuística. Se aplicó una encuesta anónima, voluntaria de preguntas cerradas, previamente validada. Para el cálculo del tamaño de la muestra se consideró una población de 189 estudiantes, frecuencia esperada de 30\%, un límite de confianza de 5\% con un nivel de confianza de 95\% arrojando como número mínimo 119 estudiantes como muestra representativa. Los datos fueron guardados en Microsoft Office Excel

\footnotetext{
1. Universidad Santa Clara de Asís. Caaguazú, Facultad de Ciencias de la Salud, Paraguay. E-mail: kukiparedes_17@hotmail.com DOI: $10.26885 /$ rcei.foro.2019.245
}

Trabajo publicado en acceso abierto bajo Licencia Creative Commons. 
2013 y se exportó al paquete de análisis estadísticos Epiinfo V7 para su análisis. Los datos obtenidos se manejaron bajo los principios de Helsinki.

Resultados: Se analizaron 127 estudiantes del 3으 al 6을 curso, de los cuales un $58 \%$ pertenecen al sexo femenino y un $25 \%$ ocupa becas. Estudiantes afirman que las situaciones que mayor estrés les ocasiona son: realización de un examen, sobrecarga académica y exámenes actuales. Apenas les produce estrés: horas de tutoría, el depender de una beca y la competitividad entre compañeros. La presión ejercida por los padres y/o tutores es un claro indicador del nivel de estrés.

Conclusión: La mayoría de los estudiantes objeto del estudio, en algún momento de su vida académica sufren estrés, esté normalmente viene provocado por falta de tiempo, realización de exámenes o por presión que soportan de algunas personas. A su vez estamos de acuerdo en que existe un foco que radie presión sobre cualquier persona, esta sufrirá una tensión que en un periodo de tiempo pueda causar estrés, debido a la consecución de la meta que los demás han fijado para nosotros.

Palabras clave: estrés académico, estudiantes universitarios, facultad médica.

\section{RefERencias}

Jaqueline, A. (2014). El estrés un problema de salud del mundo actual. Revista de Ciencias Farmacéuticas y Bioquímicas, 2(1). http://www. revistasbolivianas.org.bo/scielo.php?script=sci_arttext\&pid=S231002652014000100013\&lng=es

Labrador, F. J. (1996). El estrés. Nuevas técnicas de control. Madrid, España.

López, J., Martin, G., de León, S., Morelos, J., Pérez, V., Flores-Ramos, M. (2013). Factores que predicen depresión en estudiantes de medicina. Gaceta Médica de México, 149(6).

Márquez-Granados, K. L. (2019). Relación entre estrés académico y consumo de alcohol y tabaco en estudiantes de educación superior. Lux Médica, 14(41). https://revistas.uaa.mx/index.php/luxmedica/article/ view/2009

Montoya Vélez, L. P., Gutiérrez, J. A., Toro Isaza, B. E. (2010). Depresión en estudiantes universitarios y su asociación con el estrés académico. CES Medicina, 24(1). http://revistas.ces.edu.co/index.php/medicina/ article/view/1011 